\title{
Fractal Based Analysis of Movement Behavior in Animal Foraging
}

\author{
H Reza Namazi* \\ 167 Division Ave Brooklyn, New York 11211, USA
} *Corresponding Author: H Reza Namazi, 167 Division Ave Brooklyn, New York 11211, USA, Email:
hrnneuromap@gmail.com

\begin{abstract}
Foraging is searching for wild food resources. Foraging theory is a branch of behavioral ecology that studies the foraging behavior of animals in response to the environment, where the animal lives. The movement of animals in searching for foods is random walk that has fractal characteristics. Fractal theory is a useful approach that widely been used in analysis of random walks. Beside several works reported in fractal analysis of movement behavior of animal foraging, no work has considered the concept of memory and fractal approach simultaneously for modelling of this behavior. In this editorial paper, we will discuss the mportance of employing fractal theory in analysis of complexity, memory content and modelling of movement behavior in animal foraging. Based on the introduced concepts, we will give several outlines for future works.
\end{abstract}

\section{Fractals Theory and Complexity Analysis}

Fractal theory has been used widely for studying the scaling properties of different biological and ecological time series [1-3]. A phenomenon showinga repeating pattern at every scale is called fractal [4]. Fractals can be regular or complex [5]. In fact, fractal objects can be characterized using a scaling exponent that is called fractal dimension. Regular and complex fractals have integer and non-integer dimensions respectively [6]. In case of all fractals, the fractal dimension should satisfy the Szpilrajn inequality [7]:

$\aleph \geq D_{T}$

Where $\mathcal{X}$ and $D_{T}$ are fractal dimension and topological dimension respectively. Fractal and Euclidean geometries are related to each other in building objects by applying processes to build blocks [7]. In case of a fractal time series, all fluctuations are correlated with each other based on power law. This correlation brings the concept of memory into account [8]. In application of fractal theory, we can call the works on analysis of DNA [9], eye movement [10], EEG signal [11], bone structure [12], respiration signal [13], human stride time series [14] and face [15]. In case of fractal analysis of animal movement, several works have been reported in literatures. For instance, Etzenhouser et al. [16] analyzed the movement patterns of white-tailed deer and Spanish goats in order to test the relation between foraging movements and environmental heterogeneity. They found out that the foraging paths of goats are more complex, having greater fractal dimension. In another work, Webb et al. [17] did fractal analysis to characterize movement paths of white-tailed deer. They found that females' movement path is more complex than males and concluded that females are able to forage more intensively in a smaller area. Also, look at [1820].

\section{Memory Concept}

Memory is an important concept that is discussed in case of random walks. In employing of fractal theory for analysis of movement behavior, we can brings the Hurst exponent into analysis in order to talk about the memory content of process.

The Hurst exponent can have any value in the range of $0-1$. In case of Brownian motion that does not show any persistence in the motion, Hurst exponent has the value of $\mathrm{H}=0.5$. In this case, the process is completely random without any memory. In case $\mathrm{H}<0.5$, the process is anti-persistent. On the other hand, in case of $\mathrm{H}>$ 0.5 , the process is persistent [21-23].

Therefore it can be said in case of $\mathrm{H}=0.5$ the process does not have any memory, but in case of $\mathrm{H}=0$ or 1 , the process has highest level of 
memory. Therefore, the closer value of Hurst exponent to 0.5 , the process has less memory. For instance, a process with $\mathrm{H}=0.3$ has higher memory compared to a process with $\mathrm{H}=0.4$. Similarly, a process with $\mathrm{H}=0.9$ has higher memory compared to a process with $\mathrm{H}=0.7$.

Similarly, there has been variety of works in biology and medicine that employed Hurst exponent for analysis of random walks. Using Hurst exponent in investigation about EEG signal [24] and human gait [25] are noteworthy to mention. Very limited works have been reported in literatures that consider Hurst exponent in analysis of animal movement. MacIntosh et al. [26] employed the Hurst exponent for analysis of fractal time in binary dive sequences of little penguins. The result of their analysis showed that dive sequences are long-range dependent and persistent. Also, look at [27]. However, none of the reported works discussed about the memory aspect of Hurst exponent. Bringing the concept of memory using Hurst exponent in analysis of animal movement can solve many phenomena in their behavior of searching for foods.

\section{Modeling}

An important step after analysis of random walk is working on modelling. Modelling of random walks can be done using different approaches. During years, fractal theory has shown its importance in modelling of random walks [28]. Scientists have used different types of fractal models in order to model different processes in biology and medicine. For instance, in [8] we developed a model based on Fractional Diffusion Equations (FDE) in order to model the behavior of Electroencephalogram (EEG) signal in response to external stimuli. In another work [14], similar model has been used for modelling of Human Gait time series due to auditory stimuli. In a similar approach, we can employ different types of fractal equations in order model the animal movements in searching for foods. In this model, food is considered as external stimulus that arouse the attention of animal, and accordingly animal will do the random walk to search for it. This modelling will allow us to predict the movement of animal that is our ultimate goal. This modelling has advantages over the last reported mathematical models [29-31] that did not consider the memory concept of animal movements using Hurst exponent, which relates animal movement to its fractal dynamics. In addition, other methods such as machine learning can be applied for this purpose with considering the fractal phenomenon. For instance, a fractal deep learning neural network with a varied time delay can be developed to predict the variations of animal random walk based on fractal behavior.

\section{REFERENCES}

[1] Klonowski, W. Fractal Analysis of Electroencephalographic Time Series (EEG Signals). The Fractal Geometry of the Brain. 413-429, 2016.

[2] Ihlen, E. A. F. Introduction to Multifractal Detrended Fluctuation Analysis in Matlab. Front Physiol. 3: 141; DOI: 10.3389/fphys.2012.00141 (2012).

[3] Eke A, Herman P, Sanganahalli BG, Hyder F, Mukli P and Nagy Z (2012) Pitfalls in fractal time series analysis: fMRI BOLD as an exemplary case. Front. Physio. 3:417. doi: 10.3389/fphys.2012.00417.

[4] Spasic, S., Kalauzi, A., Grbic, G., Martac, L. \&Culic, M. Fractal analysis of rat brain activity after injury. Medical and Biological Engineering and Computing. 43 (3), 345-348 (2004).

[5] Namazi, H. and Kulish, V. V. Fractal Based Analysis of the Influence of Odorants on Heart Activity. Sci. Rep. 6, 38555; doi: 10.1038/srep38555 (2016).

[6] Long, G. \& Xu, C. The Fractal Dimensions of Complex Networks. CHIN. PHYS. LETT. 26 (8), 088901-1,4 (2009).

[7] Namazi H, Akrami A, Haghighi R, Delaviz A, Kulish V.V. Analysis of the Influence of Element's Entropy on the Bulk Metallic Glass (BMG) Entropy, Complexity and Strength. Metallurgical and Materials Transactions A.DOI: 10.1007/s11661-016-3870-3 (2016).

[8] Namazi, H. \&Kulish, V. V. Fractional Diffusion Based Modelling and Prediction of Human Brain Response to External Stimuli. Comput. Math. Methods Med.2015, 148534; DOI:10.1155/2015/148534 (2015).

[9] Sadana, A. A fractal analysis of protein to DNA binding kinetics using biosensors. BiosensBioelectron. 18 (8), 985-97 (2003).

[10] Namazi, H., Kulish, V. V. \&Akrami, A. The analysis of the influence of fractal structure of stimuli on fractal dynamics in fixational eye movements and EEG signal. Sci. Rep.6, 26639; DOI: 10.1038/srep26639 (2016).

[11] Namazi, et al. A signal processing based analysis and prediction of seizure onset in patients with epilepsy. Oncotarget7, 342-350; DOI: 10.18632/oncotarget.6341 (2016).

[12] Kyung-Hoe, H. et al. Fractal analysis of mandibular trabecular bone: optimal tile sizes 
for the tile counting method. Imaging Sci. Dent. 41, 71-78 (2011).

[13] Peng, C. K. et al. Quantifying Fractal Dynamics of Human Respiration: Age and Gender Effects. Ann. Biomed. Eng. 30 (5), 683-692 (2002).

[14] Namazi, H. \&Kulish, V. V. Mathematicalbased modeling and prediction of the effect of external stimuli on human gait. Int. J. Numer. Method Biomed. Eng.32; DOI: 10.1002/ cnm.2805 (2016).

[15] Mohamed, B., Benkkadour, M. K., Redwan, T. \&Senouci, M. A Novel Technique For Human Face Recognition Using Fractal Code and Bidimensional Subspace. CIIA 2015, IFIP AICT. 31-42, 2015.

[16] Etzenhouser, M. J., Owens, M. K., Spalinger, D. E., Murden, S. B. Foraging behavior of browsing ruminants in a heterogeneous landscape. Landscape Ecology. 13, 55-64 (1998).

[17] Webb, S. L., Riffell, S. K., Gee, K. L. \&Demarais, S. Using Fractal Analyses to Characterize Movement Paths of White-Tailed Deer and Response to Spatial Scale. Journal of Mammalogy 90, 1210-1217 (2009).

[18] Bartumeus, F. Behavioral intermittence, Le'vy patterns, and randomness in animal movement. Oikos 118: 488-494; DOI: 10.1111/j.16000706.2008.17313.x (2009).

[19] Frederic Bartumeus1 \& Levin S. A. Fractal reorientation clocks: Linking animal behavior to statistical patterns of search. PNAS. 105, 19072-19077 (2008).

[20] Cole, B. J. Fractal time in animal behaviour: the movement activity of Drosophila. AnimBehav. 50, 1317-1324 (1995).

[21] Namazi H, Kulish VV. A mathematical based calculation of a myelinated segment in axons. Comput. Biol. Med. 2013 Jul; 43(6):693-8. doi: 10.1016/j.compbiomed.2013.03.005.

[22] Seetharaman, K., Namazi, H. \&Kulsih, V. V. Phase lagging model of brain response to external stimuli-modeling of single action potential. Comput. Biol. Med. 42, 857-862 (2012).

[23] Namazi, H. \&Kulish V. V. Mathematical Modeling of Human Brain Neuronal Activity in the Absence of External Stimuli. Journal of Medical Imaging and Health Informatics 2(4):400-407 (2012)

[24] Namazi, H. et al. Analysis of the influence of memory content of auditory stimuli on the memory content of EEG signal. Oncotarget. 7(35):56120-56128. doi: 10.18632/ oncotarget. 11234 (2016).

[25] Goshvarpour, A. \& Goshvarpour, A. Nonlinear Analysis of Human Gait Signals. I.J. Information Engineering and Electronic Business, 2, 15-21 (2012).

[26] MacIntosh, A.J.J., Pelletier, L., Chiaradia, A., Kato, A. \& Ropert-Coudert, Y. Temporal fractals in seabird foraging behaviour: diving through the scales of time. Sci. Rep. 3, 1884; DOI: 10.1038/srep01884 (2013).

[27] Gutiérrez, E. D. and Cabrera, J. L. A neural coding scheme reproducing foraging trajectories. Sci. Rep. 5, 18009; doi: 10.1038/srep18009 (2015).

[28] d'Auriac, J. C. A., Benoit, A. \&Rammal, R. Random walk on fractals: numerical studies in two dimensions. J. Phys. A: Math. Gen. 16, 4039, 1983.

[29] Preisler, H. K., Ager, A. A., Johnson, B. K. \&Kie, J. G. Modeling animal movements using stochastic differential equations. Environ metrics 15, 643-657; DOI: 10.1002/ env.636 (2004).

[30] Blackwell, P. G. Random diffusion models for animal movement. 100, 87-102 (1997).

[31] Bestley, S., Jonsen, I., Harcourt, R. G., Hindell, M. A. \& Gales, N. J. Putting the behavior into animal movement modeling: Improved activity budgets from use of ancillary tag information. EcolEvol. 6, 8243-8255 (2016).

Citation: H Reza Namazi. Fractal Based Analysis of Movement Behavior in Animal Foraging. ARC Journal of Neuroscience. 2017; 2(3):1-3. doi:dx.doi.org/10.20431/2456-057X.0203001.

Copyright: (C) 2017 Authors. This is an open-access article distributed under the terms of the Creative Commons Attribution License, which permits unrestricted use, distribution, and reproduction in any medium, provided the original author and source are credited. 